This item was submitted to Loughborough's Research Repository by the author.

Items in Figshare are protected by copyright, with all rights reserved, unless otherwise indicated.

\title{
Disturbance rejection flight control for small fixed-wing unmanned aerial vehicles
}

PLEASE CITE THE PUBLISHED VERSION

http://dx.doi.org/10.2514/1.G001958

\section{PUBLISHER}

American Institute of Aeronautics and Astronautics

VERSION

AM (Accepted Manuscript)

\section{PUBLISHER STATEMENT}

This work is made available according to the conditions of the Creative Commons Attribution-NonCommercialNoDerivatives 4.0 International (CC BY-NC-ND 4.0) licence. Full details of this licence are available at: https://creativecommons.org/licenses/by-nc-nd/4.0/

\section{LICENCE}

CC BY-NC-ND 4.0

\section{REPOSITORY RECORD}

Liu, Cunjia, and Wen-Hua Chen. 2016. "Disturbance Rejection Flight Control for Small Fixed-wing Unmanned Aerial Vehicles”. figshare. https://hdl.handle.net/2134/22229. 


\title{
Disturbance rejection flight control for small
}

\section{fixed-wing unmanned aerial vehicles}

\author{
Cunjia Liu ${ }^{1}$ and Wen-Hua Chen ${ }^{2}$ \\ Loughborough University, Leicestershire, LE11 3TU, United Kingdom
}

\section{Nomenclature}

$A R \quad$ wing aspect ratio, 0.152

b wingspan, $0.28956 \mathrm{~m}$

c mean aerodynamic chord of the wing, $0.18994 \mathrm{~m}$

$C_{D} \quad$ drag coefficient

$C_{D_{0}} \quad$ drag coefficient due to parasitic drag, 0.0437

$C_{L} \quad$ lift coefficient

$C_{L_{0}} \quad$ lift coefficient at aero angle of attack, 0.28

$C_{L_{\alpha}} \quad$ lift coefficient due to angle of attack, 3.45

$C_{L_{\delta_{e}}} \quad$ lift coefficient due to elevator deflection, -0.36

$C_{m} \quad$ pitching moment coefficient,

$C_{m_{0}} \quad$ pitching moment coefficient at zero condition , -0.02338

$C_{m_{\alpha}} \quad$ pitching moment coefficient due to angle of attack, -0.38

$C_{m_{\delta_{e}}}$ pitching moment coefficient due to elevator deflection, -0.5

$C_{\text {prop }}$ mean chord of the propeller, 1.0

$D \quad$ drag, $\mathrm{N}$

$g \quad$ gravitational acceleration, $9.8 \mathrm{~m} / \mathrm{s}^{2}$

e $\quad$ Oswald efficiency factor, 0.9

$\hbar \quad$ altitude, $\mathrm{m}$

$I_{y y} \quad$ moment of inertia, $1.135 \mathrm{~kg} \cdot \mathrm{m}^{2}$

\footnotetext{
${ }^{1}$ Lecturer, Department of Aeronautical and Automotive Engineering, Loughborough University

2 Professor, Department of Aeronautical and Automotive Engineering, Loughborough University.
} 
$K_{\text {motor }}$ motor constant, 80

$L \quad$ lift, $\mathrm{N}$

$m \quad$ mass, $13.5 \mathrm{~kg}$

$M \quad$ pitch moment, $\mathrm{N} / \mathrm{m}$

$q \quad$ pitch $\mathrm{rate}, \mathrm{rad} / \mathrm{s}$

$S \quad$ reference area, $0.55 \mathrm{~m}^{2}$

$S_{\text {prop }}$, propeller swiping area, $0.2027 \mathrm{~m}^{2}$

$T \quad$ thrust, $\mathrm{N}$

$V \quad$ velocity, $\mathrm{m} / \mathrm{s}$

$w_{x}, w_{h}$ wind components, $\mathrm{m} / \mathrm{s}$

$\alpha \quad$ angle of attack, rad

$\gamma \quad$ flight path angle, rad

$\delta_{e} \quad$ elevator deflection, rad

$\delta_{T} \quad$ throttle setting, $\% / 100$

$\rho \quad$ density of air, $1.2682 \mathrm{~kg} / \mathrm{m}^{3}$

Subscript

a air-mass referenced quantity

$x \quad$ inertially referenced quantity along $\mathrm{x}$-axis direction

$h \quad$ inertially referenced quantity along h-axis direction

\section{Introduction}

Recent years have seen the unprecedented development of various unmanned aerial vehicles (UAVs) and their growing applications in both military and civilian domains. Among different types of UAVs, small UAVs (usually under $20 \mathrm{~kg}$ ) are popular flying platforms which are portable to deploy, low-cost to build but still able to carry essential payloads to carry out challenging missions like surveillance in urban environments. However, because of their relatively slow airspeed, light 
weight and very often limited power, they are also susceptible to external disturbances, such as wind gusts and local wake of buildings, which may deteriorate the flight performance or even cause serious problems on stability and flight quality. This presents a challenge on flight control design for this kind of aircraft, because not only the system nonlinearities and uncertainties need to be deal with, but also the effects from unknown disturbances need to be considered.

Wind influences on flight dynamics of small fixed-wing UAVs have been investigated by many previous studies. Wind characteristics and their implications on micro UAV have been recorded and analyzed in [I]. Different solutions for UAV path-following in wind have been developed, for example in [2-4] , aiming to improve the tracking accuracy. In [5], the flight control design of a UAV in stochastic winds is performed by using a smooth approximation of a sliding mode controller. Optimal flight control for more general aircraft under severe weather conditions has been considered in [6], where the formulated stochastic control has been solved by a polynomial chaos method.

This work aims to improve the flight performance of small fixed-wing UAVs in wind conditions by designing an advanced flight controller based on the available aircraft model. In particular, the control design focuses on the longitudinal dynamic model, because it is important for taking off and landing of such a UAV in the presence of wind disturbances [6, []]. General longitudinal aircraft dynamics possess strong nonlinearities and uncertainties, which has necessitated the use of nonlinear control methods. Robust nonlinear dynamic inversion (NDI) has been applied to control the longitudinal dynamics of a hypersonic aircraft [8]. An adaptive sliding mode control was developed later to tackle the same problem [9], which also considered the case that only a part of the aircraft states is measurable. In recent years, robust adaptive control techniques have been applied to the longitudinal channel of air-breathing hypersonic vehicles with flexible structures and non-minimum phase behaviors [10, ㅍ]. In terms of small UAVs, reference [12] has proposed a novel adaptive backstepping method to tackle the system uncertainties as well as thrust saturation.

Comparing to preceding flight control designs, the problem considered in this work encounters a different challenge, which is the significance of wind disturbance on the flight dynamics of small UAVs. The varying wind disturbances can easily get close to the operational airspeed of a UAV, hence instead of being treated as uncertainties, they should be explicitly considered. To this end, this 
note advocates the disturbance observer based control (DOBC) technique (see [13] for an overview) to suppress the adverse impacts of wind. The philosophy of this method is first to quickly estimate the wind components based on their influences on flight dynamics using a nonlinear disturbance observer, and then to exploit this information in flight control design to compensate for the wind by actively deploying control surfaces. Previous studies have shown the benefits of this control strategy in flight control, for example on missiles [14, [5], hypersonic vehicles [16] and small unmanned helicopters [177, 118].

To improve the gust alleviation of small fixed-wing UAVs, this note first investigates the aircraft dynamics under wind disturbances, which will be used in the disturbance observer design. Next, a novel nonlinear disturbance observer is developed for this particular UAV model with wind influences. Comparing to previous DOBC designs (e.g. [14-[8]) where disturbances are lumped together and assumed to be directly added on the state dynamics, a key feature of this work is that the various disturbances acting on the aircraft are explicitly modeled and their couplings with system states are appropriately handled in the disturbance observer design. This nontrivial design can guarantee that the stability property of the observer is decoupled from the system states. On the other hand, to establish the baseline control, a NDI method is adopted and the disturbance estimates are incorporated by designing a compensation control gain. The overall structure of the DOBC design is rather explicit, where the compensation control effort based on disturbance estimates can be treated as a "patch" to the baseline controller.

The remaining parts of this note are organized as follows. In Section $\mathbb{W}$, the aircraft longitudinal dynamics under wind disturbances are introduced. Next, Section $\mathbb{W}$ details the development of the nonlinear disturbance observer, which is followed by disturbance rejection flight control in Section ㅍ․ Simulation studies are given in Section $\square$, whereas the conclusions are provided in Section $\nabla$.

\section{UAV dynamic model subject to wind disturbance}

This section introduces the longitudinal flight dynamics of a small fixed-wing UAV under wind disturbances. The coordinates of the aircraft and axes of wind are given in Fig.⿴囗. Following [1.9, 20], 
the dynamic equations can be expressed as follows:

$$
\begin{aligned}
& \dot{x}=V_{a} \cos \gamma_{a}+w_{x} \\
& \dot{\hbar}=V_{a} \sin \gamma_{a}+w_{h} \\
& \dot{V}_{a}=\frac{(T+\Delta T) \cos \alpha-(D+\Delta D)}{m}-g \sin \gamma_{a}-\cos \gamma_{a} \dot{w}_{x}-\sin \gamma_{a} \dot{w}_{h} \\
& \dot{\gamma}_{a}=\frac{(T+\Delta T) \sin \alpha+(L+\Delta L)}{m V_{a}}-\frac{g \cos \gamma_{a}}{V_{a}}+\frac{\sin \gamma_{a}}{V_{a}} \dot{w}_{x}-\frac{\cos \gamma_{a}}{V_{a}} \dot{w}_{h} \\
& \dot{\alpha}=q-\dot{\gamma}_{a} \\
& \dot{q}=\frac{1}{I_{y y}}(M+\Delta M)
\end{aligned}
$$

where the external lift, drag, thrust and pitch moment are functions of aircraft parameters, states and control inputs, which can be calculated as

$$
\begin{aligned}
L & =\frac{1}{2} \rho V_{a}^{2} S C_{L} \\
D & =\frac{1}{2} \rho V_{a}^{2} S C_{D} \\
T & =\frac{1}{2} \rho S_{\text {prop }} C_{\text {prop }}\left(\left(K_{\text {motor }} \delta_{T}\right)^{2}-V_{a}^{2}\right) \\
M & =\frac{1}{2} \rho V_{a}^{2} S c C_{m}
\end{aligned}
$$

The aerodynamic coefficients can be further expressed as

$$
\begin{aligned}
& C_{L}=C_{L_{0}}+C_{L_{\alpha}} \alpha+C_{L_{\delta_{e}}} \delta_{e} \\
& C_{D}=C_{D_{p}}+\frac{\left(C_{L_{0}}+C_{L_{\alpha}} \alpha\right)^{2}}{\pi e A R}+C_{D_{\delta_{e}}} \delta_{e} \\
& C_{m}=C_{m_{0}}+C_{m_{\alpha}} \alpha+C_{m_{\delta_{e}}} \delta_{e}
\end{aligned}
$$

To account for any uncertainties or unmodeled factors in external forces, the uncertainty terms $\Delta L, \Delta D, \Delta T$ and $\Delta M$ for the lift, drag, thrust and pitch moment, respectively, are also incorporated into the UAV model. To facilitate the control design, the engine dynamic model is assumed to take a second-order form such that

$$
\ddot{\delta}_{T}=k_{1} \dot{\delta}_{T}+k_{2} \delta_{T}+k_{3} \delta_{t}
$$

where $\delta_{t}$ is the actual throttle setting. For the sake of simplicity, this study chooses $k_{1}=k_{2}=0$ and $k_{3}=1$. This dynamic extension also ensures that the relative degree is well-defined in the control design $[8]$. 


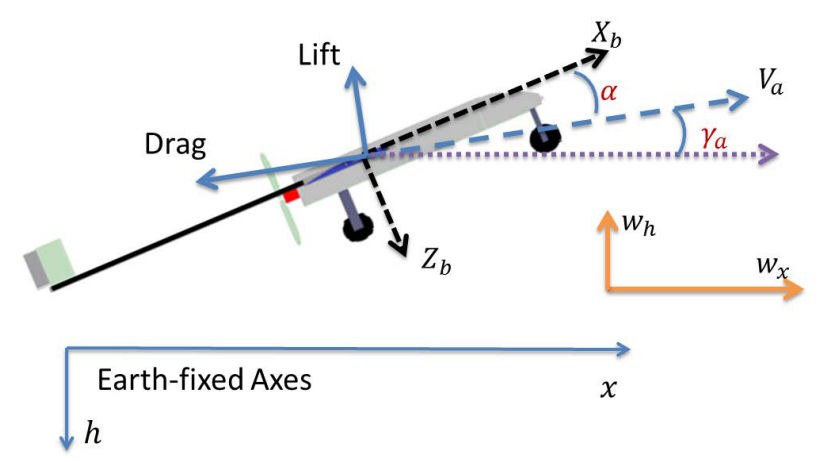

Fig. 1 Illustration of system coordinates, angles and aerodynamics forces

The wind influences on UAV dynamics can be observed from model (四). The wind velocities $w_{x}$ and $w_{h}$ have direct influences on aircraft states $x$ and $\hbar$, respectively, whereas the wind accelerations $\dot{w}_{x}$ and $\dot{w}_{h}$ can affect the dynamics of airspeed $V_{a}$ and flight path angle $\gamma_{a}$. Given the relatively low airspeed of small UAVs, the wind velocities and accelerations may have significant impact on the aircraft performance. It can also been seen from (四) that the steady winds only affect the first two states, but wind gusts or wind shears with non-zero $\dot{w}_{x}$ and $\dot{w}_{h}$ influence the flight dynamics, especially the airspeed $V_{a}$, which is critical in determining aerodynamics forces and torques applied on the aircraft.

The task of flight control design is to form a feedback loop to maintain the aircraft states and/or to drive some of the outputs (e.g. airspeed and altitude) to specified values. In conventional feedback control design, since the wind components and the force/moment disturbances are unknown to the controller, the actual control performance will be degraded because of their adverse effects. To improve flight performance for small UAVs in windy conditions, this study adopts a two-step approach to take into account the disturbances in flight control design. Specifically, unknown disturbances are first estimated based on their effects on the nominal UAV dynamics. Then, those estimates are incorporated in control design to compensate the influences due to disturbances.

To facilitate the control design, the force and moment disturbances, which usually have different 
characteristics from wind acceleration terms, are lumped together such that

$$
\begin{aligned}
& d_{D}=(\Delta T \cos \alpha-\Delta D) / m \\
& d_{L}=(\Delta T \sin \alpha+\Delta L) / m \\
& d_{M}=\Delta M / I_{y y}
\end{aligned}
$$

Therefore, the system dynamical equations (四) together with engine dynamics (四) can be expressed compactly as

$$
\dot{\mathbf{x}}=\mathbf{f}(\mathbf{x})+\mathbf{g}(\mathbf{x}) \mathbf{u}+\mathbf{s}(\mathbf{x}) \mathbf{d}
$$

where the state is defined as $\mathbf{x}=\left[\begin{array}{llllllll}x & \hbar & V_{a} & \gamma_{a} & \alpha & q & \delta_{T} & \dot{\delta}_{T}\end{array}\right]^{T}$, the control input is $\mathbf{u}=\left[\begin{array}{ll}\delta_{e} & \delta_{t}\end{array}\right]^{T}$ and the disturbance vector is defined as $\mathbf{d}=\left[\begin{array}{lllllll}w_{x} & w_{h} & \dot{w}_{x} & \dot{w}_{h} & d_{D} & d_{L} & d_{M}\end{array}\right]^{T}$. The system output chosen to be controlled is $\mathbf{y}=\mathbf{h}(\mathbf{x})=\left[\begin{array}{ll}\hbar & V_{a}\end{array}\right]^{T}$.

\section{Disturbance observer design}

The objective of this section is to develop a disturbance observer to estimate the wind components as well as the lumped force and moment disturbances that are applied to the nominal longitudinal dynamics of a UAV. The generated estimates will be incorporated subsequently in control design to compensate for their effects.

To design the disturbance observer, the properties of the disturbance terms are investigated first. The dynamics of the disturbance terms can be captured by the following equation

$$
\dot{\mathbf{d}}=\underbrace{\left[\begin{array}{ccc}
\mathbf{0}_{2 \times 2} & \mathbf{I}_{2} & \mathbf{0}_{2 \times 2} \\
\mathbf{0}_{4 \times 2} & \mathbf{0}_{4 \times 2} & \mathbf{0}_{4 \times 2} \\
\hdashline \mathbf{0}_{1 \times 2} & \mathbf{0}_{1 \times 2} & \mathbf{0}_{1 \times 2}
\end{array}\right]}_{\mathbf{A}} \mathbf{d}+\mathbf{w}=\left[\begin{array}{c}
\mathbf{A}_{1} \\
\hdashline \overrightarrow{\mathbf{A}}_{2}
\end{array}\right] \mathbf{d}+\mathbf{w}
$$

where $\mathbf{w}=\left[\begin{array}{lllllll}0 & 0 & \ddot{w}_{x} & \ddot{w}_{h} & \dot{d}_{D} & \dot{d}_{L} & \dot{d}_{M}\end{array}\right]^{T}$. Given the nature of atmospheric wind, the assumption that the derivatives of wind accelerations are bounded is adopted in this work, so that $\left\|\ddot{w}_{(\cdot)}\right\|<\sigma_{w}$, where $\sigma_{w}$ is a positive constant. It can be further assumed that $\lim _{t \rightarrow \infty} \ddot{w}_{(\cdot)} \approx 0$, which means that the wind acceleration will settle eventually. On the other hand, the force and moment disturbances are commonly assumed to be slowly varying with respect to the observer dynamics [3, [6, [7]], i.e. $\dot{d}_{D} \approx 0, \dot{d}_{L} \approx 0$ and $\dot{d}_{M} \approx 0$, since the observer dynamics can be tuned to be fast. 
The wind accelerations and the force disturbances may have different characteristics, however they are coupled together and applied on the same channels as shown in (एव) and (एत), respectively. Therefore, it is difficult to distinguish their effects and to estimate their respective values merely based on their impacts on those states. A common solution in disturbance observer design is to lump them together and consider their combined influences. However, this means to discard the internal dynamics of the wind disturbance, which in turn will degrade the estimation performance and require high gains in the observer [21]. To solve this problem, a novel disturbance observer design is developed in this work by exploiting extra information that can be provided by the UAV sensor suit. In this case, ground speed measurements provided by GPS are adopted in addition to the state used in (四). Define $\dot{x}=V_{x}$ and $\dot{\hbar}=V_{h}$. The dynamics of these two states can be obtained

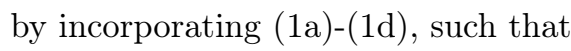

$$
\begin{aligned}
& \dot{V}_{x}=\frac{T \cos \alpha-D}{m} \cos \gamma_{a}-\frac{T \sin \alpha+L}{m} \sin \gamma_{a}+d_{D} \cos \gamma_{a}-d_{L} \sin \gamma_{a} \\
& \dot{V}_{h}=\frac{T \cos \alpha-D}{m} \sin \gamma_{a}+\frac{T \sin \alpha+L}{m} \cos \gamma_{a}-g+d_{D} \sin \gamma_{a}+d_{L} \cos \gamma_{a}
\end{aligned}
$$

Note that the disturbance $d_{M}$ acting on the pitch channel (田) is decoupled from other channels. Therefore, the disturbance observer for $d_{M}$ can be designed by following the standard process (see e.g. [16]). For the sake of simplicity, the disturbance observer design in this note only focuses on the coupled wind components and force disturbances.

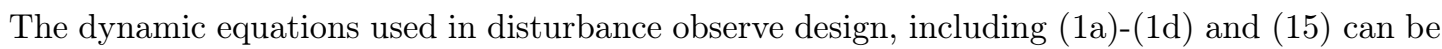
expressed in a general compact form

$$
\dot{\overline{\mathbf{x}}}=\mathbf{f}_{1}(\overline{\mathbf{x}})+\mathbf{g}_{1}(\overline{\mathbf{x}}) \mathbf{u}+\mathbf{s}_{1}(\overline{\mathbf{x}}) \mathbf{d}_{1}
$$

where the augmented state is defined as $\overline{\mathbf{x}}=\left[\begin{array}{llllll}x & \hbar & V_{a} & \gamma_{a} & V_{x} & V_{h}\end{array}\right]^{T}$, the disturbance vector is 
$\mathbf{d}_{1}=\left[\begin{array}{llllll}w_{x} & w_{h} & \dot{w}_{x} & \dot{w}_{h} & d_{D} & d_{L}\end{array}\right]^{T}$ and the disturbance input matrix $s_{1}(\overline{\mathbf{x}})$ has the following structure

$$
\mathbf{s}_{1}(\overline{\mathbf{x}})=\left[\begin{array}{cccccc}
1 & 0 & 0 & 0 & 0 & 0 \\
0 & 1 & 0 & 0 & 0 & 0 \\
0 & 0 & -\cos \gamma_{a} & -\sin \gamma_{a} & 1 & 0 \\
0 & 0 & \sin \gamma_{a} / V_{a} & -\cos \gamma_{a} / V_{a} & 0 & 1 / V_{a} \\
0 & 0 & 0 & 0 & \cos \gamma_{a} & -\sin \gamma_{a} \\
0 & 0 & 0 & 0 & \sin \gamma_{a} & \cos \gamma_{a}
\end{array}\right]
$$

The nonlinear disturbance observer adopted in this note follows the structure developed in [22], which can be expressed as

$$
\begin{aligned}
& \dot{\mathbf{z}}=\left[\mathbf{A}_{1}-\mathbf{l}(\overline{\mathbf{x}}) \mathbf{s}_{1}(\overline{\mathbf{x}})\right] \mathbf{z}+\mathbf{A}_{1} \mathbf{p}(\overline{\mathbf{x}})-\mathbf{l}(\overline{\mathbf{x}})\left[\mathbf{s}_{1}(\overline{\mathbf{x}}) \mathbf{p}(\overline{\mathbf{x}})+\mathbf{f}_{1}(\overline{\mathbf{x}})+\mathbf{g}_{1}(\overline{\mathbf{x}}) \mathbf{u}\right] \\
& \hat{\mathbf{d}}_{1}=\mathbf{z}+\mathbf{p}(\overline{\mathbf{x}})
\end{aligned}
$$

where $\mathbf{z}$ is the internal state of the observer and $\hat{\mathbf{d}}_{1}$ is the estimated disturbance vector. The nonlinear function $\mathbf{p}(\overline{\mathbf{x}})$ needs to be designed based on the system equations and then the observer gain $\mathbf{l}(\overline{\mathbf{x}})$ can be determined by

$$
\mathbf{l}(\overline{\mathbf{x}})=\frac{\partial \mathbf{p}(\overline{\mathbf{x}})}{\partial \overline{\mathbf{x}}}
$$

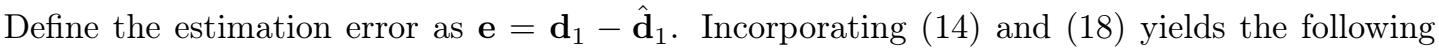
error dynamics

$$
\begin{aligned}
\dot{\mathbf{e}} & =\mathbf{A}_{1} \mathbf{d}_{1}+\mathbf{w}_{1}-\left(\dot{\mathbf{z}}+\frac{\partial \mathbf{p}(\overline{\mathbf{x}})}{\partial \overline{\mathbf{x}}} \dot{\overline{\mathbf{x}}}\right) \\
& =\left[\mathbf{A}_{1}-\mathbf{l}(\overline{\mathbf{x}}) \mathbf{s}_{1}(\overline{\mathbf{x}})\right] \mathbf{e}+\mathbf{w}_{1}
\end{aligned}
$$

where $\mathbf{w}_{1}=\left[\begin{array}{cccccc}0 & 0 & \ddot{w}_{x} & \ddot{w}_{h} & \dot{d}_{D} & \dot{d}_{L}\end{array}\right]^{T}$. Assuming the derivatives of the disturbances $\mathbf{w}_{1}$ are bounded, it can be shown that the disturbance error dynamics (ख्य) is input-to-state (ISS) stable if the observer gain is chosen such that

$$
\dot{\mathbf{e}}=\left[\mathbf{A}_{1}-\mathbf{l}(\overline{\mathbf{x}}) \mathbf{s}_{1}(\overline{\mathbf{x}})\right] \mathbf{e}
$$

is asymptotically stable [1]3]. However, it is a challenging task to find the nonlinear function $\mathbf{p}(\overline{\mathbf{x}})$

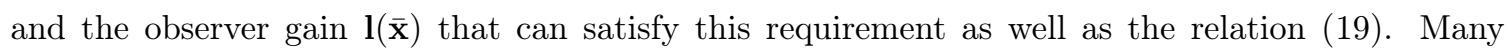


previous designs exploit the simple structure of disturbance input matrix $\mathbf{s}_{1}(\overline{\mathbf{x}})$ being a identity matrix so that the observer gain can be chosen as a constant matrix and $\mathbf{p}(\overline{\mathbf{x}})$ can be found by integration (see e.g. [[6], [7, [2:3]). Unfortunately, this method cannot be adopted in this application given $\mathbf{s}_{1}(\overline{\mathbf{x}})$ defined in $(\mathbb{\square})$.

To tailor the general disturbance observer design for the UAV longitudinal model, a novel observer gain structure is developed by designing the nonlinear function $\mathbf{p}(\overline{\mathbf{x}})$ as

$$
\mathbf{p}(\overline{\mathbf{x}})=\left[\begin{array}{c}
l_{1} \cdot x \\
l_{2} \cdot h \\
-l_{3} \cdot V_{a} \cos \gamma_{a}+l_{3} \cdot V_{x} \\
-l_{4} \cdot V_{a} \sin \gamma_{a}+l_{4} \cdot V_{h} \\
l_{5} \cdot V_{a}-V_{a} \cos \gamma_{a}+V_{x} \\
l_{6} \cdot V_{a} \gamma_{a}-V_{a} \sin \gamma_{a}+V_{h}
\end{array}\right]
$$

where $l_{i}>0, i=1, \ldots, 6$, are gain parameters to be tuned. Correspondingly, the observer gain matrix can be calculated as

$$
\mathbf{l}(\overline{\mathbf{x}})=\left[\begin{array}{cccccc}
l_{1} & 0 & 0 & 0 & 0 & 0 \\
0 & l_{2} & 0 & 0 & 0 & 0 \\
0 & 0 & -l_{3} \cdot \cos \gamma_{a} & l_{3} \cdot V_{a} \sin \gamma_{a} & l_{3} & 0 \\
0 & 0 & -l_{4} \cdot \sin \gamma_{a} & -l_{4} \cdot V_{a} \cos \gamma_{a} & 0 & l_{4} \\
0 & 0 & l_{5}-\cos \gamma_{a} & V_{a} \sin \gamma_{a} & 1 & 0 \\
0 & 0 & l_{6} \cdot \gamma_{a}-\sin \gamma_{a} & \left(l_{6}-\cos \gamma_{a}\right) V_{a} & 0 & 1
\end{array}\right]
$$

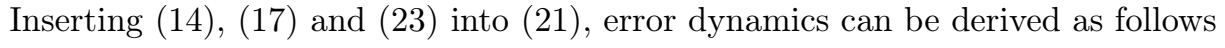

$$
\dot{\mathbf{e}}=\left[\begin{array}{c}
\dot{e}_{1} \\
\dot{e}_{2} \\
\dot{e}_{3} \\
\dot{e}_{4} \\
\dot{e}_{5} \\
\dot{e}_{6}
\end{array}\right]=\underbrace{\left[\begin{array}{cccccc}
-l_{1} & 0 & 1 & 0 & 0 & 0 \\
0 & -l_{2} & 0 & 1 & 0 \\
0 & 0 & -l_{3} & 0 & 0 \\
0 & 0 & 0 & -l_{4} & -l_{5} & 0 \\
0 & 0 & l_{5} \cdot \cos \gamma_{a}-1 & l_{5} \cdot \sin \gamma_{a} & 0 \\
0 & 0 & -l_{6} \cdot\left(\sin \gamma_{a}-\gamma_{a} \cos \gamma_{a}\right) & l_{6} \cdot \cos \gamma_{a}+l_{6} \cdot \gamma_{a} \sin \gamma_{a}-1 & -l_{6} \gamma_{a} & -l_{6}
\end{array}\right]}_{\mathbf{E}} \mathbf{e}
$$


It can be seen that although the function $\mathbf{p}(\overline{\mathbf{x}})$ and observer gain matrix $\mathbf{l}(\overline{\mathbf{x}})$ are in nonlinear forms, the error dynamics (24) actually follows a linear form with relatively simple structure. Moreover, it can be verified that the eigenvalues of the estimation error dynamics are constants, or more specifically eig $(\mathbf{E})=\left\{-l_{i}\right\}, i=1, \ldots, 6$. Therefore, by choosing the gain parameters $l_{i}$, the convergence rate of disturbance estimates can be intuitively adjusted regardless of state $\overline{\mathbf{x}}$. This is a very promising feature when tuning the disturbance observer in practice. However, this is not adequate to prove the stability of the error dynamics since it is a linear-time-variant system. Proving the stability of a linear-time-varying system is very challenging in general, but by exploiting the structure of (24), the following theorem can be drawn.

Theorem 1. Assume that the flight path angle of the $U A V$ is bounded, i.e. $\gamma_{a} \leq \sigma_{\gamma}$, where $\sigma_{\gamma}$ is a

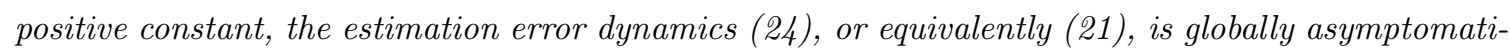
cally stable $(G A S)$ at the equilibrium of zero if the observer gain matrix follows the structure defined in (2:

Proof. Define $\boldsymbol{\epsilon}_{1}=\left[\begin{array}{ll}e_{5} & e_{6}\end{array}\right]^{T}$ and $\boldsymbol{\epsilon}_{2}=\left[\begin{array}{llll}e_{1} & e_{2} & e_{3} & e_{4}\end{array}\right]^{T}$. The error dynamics (四) can be rewritten as a cascaded system such that

$$
\begin{aligned}
& \mathcal{S}_{1}: \dot{\boldsymbol{\epsilon}}_{1}=\mathbf{F}_{1} \boldsymbol{\epsilon}_{1}+\mathbf{G}_{1} \boldsymbol{\epsilon}_{2} \\
& \mathcal{S}_{2}: \dot{\boldsymbol{\epsilon}}_{2}=\mathbf{F}_{2} \boldsymbol{\epsilon}_{2}
\end{aligned}
$$

where

$$
\mathbf{F}_{1}=\left[\begin{array}{cc}
-l_{5} & 0 \\
-l_{6} \gamma_{a} & -l_{6}
\end{array}\right], \quad \mathbf{G}_{1}=\left[\begin{array}{cccc}
0 & 0 & l_{5} \cdot \cos \gamma_{a}-1 & l_{5} \cdot \sin \gamma_{a} \\
0 & 0 & -l_{6} \cdot\left(\sin \gamma_{a}-\gamma_{a} \cos \gamma_{a}\right) & l_{6} \cdot\left(\cos \gamma_{a}+\gamma_{a} \sin \gamma_{a}\right)-1
\end{array}\right]
$$

and

$$
\mathbf{F}_{2}=\operatorname{diag}\left\{-l_{1},-l_{2},-l_{3},-l_{4}\right\}
$$

The sketch of the proof is first to show the upper system $\mathcal{S}_{1}$ is GAS when $\epsilon_{2}=0$ and input-statestable when considering $\boldsymbol{\epsilon}_{2}$ as the input. Then, if the lower system $\mathcal{S}_{2}$ is also GAS, the conclusion can be drawn by following the work of Isidori [24, Corollary 10.3.2].

Consider a Lyapunov function $V\left(\boldsymbol{\epsilon}_{1}\right)=\frac{1}{2} \boldsymbol{\epsilon}_{1}^{T} \mathbf{P}_{1} \boldsymbol{\epsilon}_{1}$, where $\mathbf{P}_{1}=\operatorname{diag}\left\{p_{1}, p_{2}\right\}$ is a positive definite matrix. Differentiating $V\left(\boldsymbol{\epsilon}_{1}\right)$ with respect to time along the trajectory of $\dot{\boldsymbol{\epsilon}}_{1}=\mathbf{F}_{1} \boldsymbol{\epsilon}_{1}$ and using 
Young's inequality gives

$$
\begin{aligned}
\dot{V} & =-p_{1} l_{5} e_{5}^{2}-p_{2} l_{6} e_{6}^{2}-p_{2} l_{6} \gamma_{a} e_{5} e_{6} \\
& \leq-p_{1} l_{5} e_{5}^{2}-p_{2} l_{6} e_{6}+p_{2} l_{6} \sigma_{\gamma}\left\|e_{5}\right\|\left\|e_{6}\right\|^{2} \\
& \leq-\left(p_{1} l_{5}-\frac{1}{2} p_{2} l_{6} \sigma_{\gamma} \kappa\right) e_{5}^{2}-\left(p_{2} l_{6}-\frac{1}{2 \kappa} p_{2} l_{2} \sigma_{\gamma}\right) e_{6}^{2}
\end{aligned}
$$

where $\kappa$ is a positive constant. By choosing $\kappa>\sigma_{\gamma} / 2$ and weighting matrix $\mathbf{P}_{1}$ such that $p_{1}>$ $\frac{1}{2 l_{5}} q_{2} l_{6} \sigma_{\gamma} \kappa$, it can be verified that $\dot{V} \leq 0$ and $\dot{V}=0$ only when $\left\|\boldsymbol{\epsilon}_{1}\right\|=0$. Therefore, the system $\mathcal{S}_{1}$ is GAS when input $\epsilon_{2}$ is zero.

Next, given the flight path angle is bounded, i.e. $\left\|\gamma_{a}\right\| \leq \sigma_{\gamma}$, it can be verified that $\left\|\mathbf{G}_{1}\right\| \leq \sigma_{G_{1}}$, where $\sigma_{G_{1}}$ is a positive constant. Therefore, the time-derivative of $V\left(\boldsymbol{\epsilon}_{1}\right)$ along the trajectory of system $\mathcal{S}_{1}$ can be rewritten from $(2 \mathrm{Wg})$ as

$$
\begin{aligned}
\dot{V} & =\boldsymbol{\epsilon}_{1}^{T}\left(\mathbf{P}_{1} \mathbf{F}_{1}\right) \boldsymbol{\epsilon}_{1}+\boldsymbol{\epsilon}_{1}^{T} \mathbf{P}_{1} \mathbf{G}_{1} \boldsymbol{\epsilon}_{2} \\
& \leq-\boldsymbol{\epsilon}_{1}^{T} \mathbf{Q} \boldsymbol{\epsilon}_{1}+\left\|\boldsymbol{\epsilon}_{1}\right\|\left\|\mathbf{P}_{1}\right\|\left\|\mathbf{G}_{1}\right\|\left\|\boldsymbol{\epsilon}_{2}\right\| \\
& \leq-\sigma_{Q}\left\|\boldsymbol{\epsilon}_{1}\right\|^{2}+\sigma_{P} \sigma_{G_{1}}\left\|\boldsymbol{\epsilon}_{1}\right\|\left\|\boldsymbol{\epsilon}_{2}\right\|
\end{aligned}
$$

where $\mathbf{Q}=\operatorname{diag}\left\{\left(q_{1} l_{5}-\frac{1}{2} q_{2} l_{6} \sigma_{\gamma} \kappa\right),\left(q_{2} l_{6}-\frac{1}{2 \kappa} q_{2} l_{2} \sigma_{\gamma}\right)\right\}, \sigma_{Q}=\lambda_{\max }(\mathbf{Q})$ and $\sigma_{P}=\lambda_{\max }(\mathbf{P})$. For any $0<\mu<1$, the foregoing inequality can be written as

$$
\dot{V} \leq-\sigma_{Q}(1-\mu)\left\|\boldsymbol{\epsilon}_{1}\right\|^{2}-\sigma_{Q} \mu\left\|\boldsymbol{\epsilon}_{1}\right\|^{2}+\sigma_{P} \sigma_{G_{1}}\left\|\boldsymbol{\epsilon}_{1}\right\|\left\|\boldsymbol{\epsilon}_{2}\right\|
$$

It follows $\dot{V} \leq-\sigma_{Q}(1-\mu)\left\|\boldsymbol{\epsilon}_{1}\right\|^{2}, \forall\left\|\boldsymbol{\epsilon}_{1}\right\|>\frac{\sigma_{P} \sigma_{G_{1}}}{\sigma_{Q} \mu}\left\|\boldsymbol{\epsilon}_{2}\right\|$. Therefore, the system $\mathcal{S}_{1}$ is input-to-state stable. At last, the globally asymptotic stability of the lower system $\mathcal{S}_{2}$ can be guaranteed if $\mathbf{F}_{2}$ is Hurwitz. Therefore, it can be concluded that the cascaded system is GAS [24, Corollary 10.3.2].

Recalling the estimation error dynamics (미) where the derivatives of the disturbances are bounded, Theorem $\square$ suggests that the proposed nonlinear function ([2]) and gain matrix ([2:3) can guarantee the ISS property of the estimation error dynamics. Moreover, given the assumption that $\lim _{t \rightarrow \infty} \ddot{W} \approx 0$, the disturbance estimate $\hat{\mathbf{d}}_{1}$ can asymptotically converge to the disturbance $\mathbf{d}_{1}$ [ए6]. It can also be observed that the stability property of the error dynamic is decoupled from the system state $\overline{\mathbf{x}}$ in the proposed disturbance observer design. This means that the disturbance observer and baseline controller can be designed separately and integrated later in the DOBC scheme. 


\section{Flight control design for disturbance rejection}

The objective of flight control is to enforce the UAV's output $\mathbf{y}$ to track the desired airspeed and altitude commands, denoted as $\mathbf{r}=\left[\begin{array}{ll}r_{1} & r_{2}\end{array}\right]^{T}$, in the presence of wind and other disturbances. To this end, a NDI controller is first designed based on the nominal UAV model to provide a baseline control. Then, the estimated disturbances are taken into account by designing the compensation control effort to eliminate their adverse effects on UAV outputs.

Given the interested outputs and the UAV dynamics in the absence of disturbance terms, the new system variables can be defined using Lie derivatives

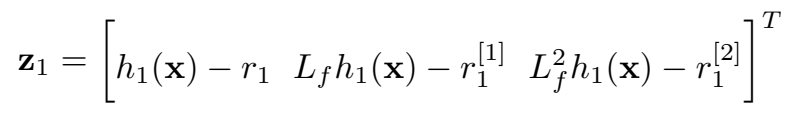

and

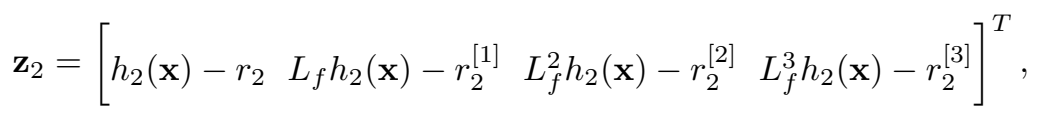

which represent the tracking errors of airspeed and altitude and their derivatives. The corresponding

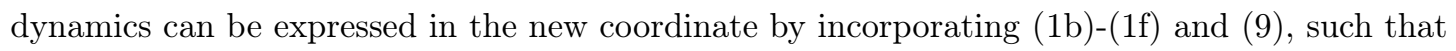

$$
\dot{\mathbf{z}}_{i}=\left[\begin{array}{c}
\dot{z}_{1}^{i} \\
\dot{z}_{2}^{i} \\
\vdots \\
\dot{z}_{\sigma_{i}}^{i}
\end{array}\right]=\left[\begin{array}{c}
z_{2}^{i} \\
z_{3}^{i} \\
\vdots \\
L_{f}^{\sigma_{i}} h_{i}(\mathbf{x})
\end{array}\right]+\left[\begin{array}{c}
0 \\
0 \\
\vdots \\
L_{g} L_{f}^{\sigma_{i}-1} h_{i}(\mathbf{x})
\end{array}\right] \mathbf{u}+\left[\begin{array}{c}
L_{s} h_{i}(\mathbf{x}) \\
L_{s} L_{f} h_{i}(\mathbf{x}) \\
\vdots \\
L_{s} L_{f}^{\sigma_{i}-1} h_{i}(\mathbf{x})
\end{array}\right] \mathbf{d}
$$

where $i \in\{1,2\}$ and the input relative degree $\sigma_{i}$ for two output channels can be calculated as $\sigma_{1}=3$ and $\sigma_{2}=4$, respectively. Collecting the dynamics equations of $z_{3}^{1}$ and $z_{4}^{2}$ gives

$$
\left[\begin{array}{c}
\dot{z}_{3}^{1} \\
\dot{z}_{4}^{2}
\end{array}\right]=\underbrace{\left[\begin{array}{c}
L_{f}^{3} h_{1}(\mathbf{x}) \\
L_{f}^{4} h_{2}(\mathbf{x})
\end{array}\right]}_{\underline{\mathbf{f}}(\mathbf{x})}+\underbrace{\left[\begin{array}{c}
L_{g} L_{f}^{2} h_{1}(\mathbf{x}) \\
L_{g} L_{f}^{3} h_{2}(\mathbf{x})
\end{array}\right]}_{\underline{\mathbf{G}}(\mathbf{x})} \mathbf{u}+\left[\begin{array}{c}
L_{s} L_{f}^{2} h_{1}(\mathbf{x}) \\
L_{s} L_{f}^{3} h_{2}(\mathbf{x})
\end{array}\right] \mathbf{d}
$$

Therefore, the transformed dynamics can be decoupled into an integrator form by defining the feedback control law in the following form

$$
\mathbf{u}=[\underline{\mathbf{G}}(\mathbf{x})]^{-1}[\mathbf{v}-\underline{\mathbf{f}}(\mathbf{x})]
$$

where $\mathbf{v}$ is the pseudo control input. To guarantee the tracking stability and reject the disturbances, this control input can be constructed by combining two parts, namely the baseline control and 
compensation control inputs, such that

$$
\mathbf{v}=\left[\begin{array}{l}
v_{1} \\
v_{2}
\end{array}\right]=\underbrace{\left[\begin{array}{cccccc}
-k_{1}^{1}, & \cdots & -k_{3}^{1} & 0 & \cdots & 0 \\
0 & \cdots & 0 & -k_{1}^{2}, & \cdots & -k_{4}^{2}
\end{array}\right]}_{\mathbf{K}}\left[\begin{array}{c}
\mathbf{z}_{1} \\
\mathbf{z}_{2}
\end{array}\right]+\underbrace{\left[\begin{array}{c}
\lambda_{1}(\mathbf{x}) \\
\lambda_{2}(\mathbf{x})
\end{array}\right]}_{\boldsymbol{\Lambda}(\mathbf{x})} \hat{\mathbf{d}}
$$

where the control gain matrix $\mathbf{K}$ can be designed based on the nominal dynamics in the absence of disturbances and the compensation control efforts $\boldsymbol{\Lambda}(\mathbf{x})$ used in this work follows the suggestion in [2.5], such that

$$
\lambda_{1}(\mathbf{x})=-k_{1}^{1} L_{s} h_{1}(\mathbf{x})-k_{2}^{1} L_{s} L_{f} h_{1}(\mathbf{x})-L_{s} L_{f}^{2} h_{1}(\mathbf{x})
$$

and

$$
\lambda_{2}(\mathbf{x})=-k_{1}^{2} L_{s} h_{2}(\mathbf{x})-k_{2}^{2} L_{s} L_{f} h_{2}(\mathbf{x})-k_{3}^{2} L_{s} L_{f}^{2} h_{2}(\mathbf{x})-L_{s} L_{f}^{3} h_{2}(\mathbf{x})
$$

By inserting the pseudo control ([7) and the actual control ([6]) into the transformed system dynamics (34), the closed-loop dynamics can be expressed in the following form

$$
\dot{\mathbf{z}}_{i}=\underbrace{\left[\begin{array}{cccc}
0 & 1 & \cdots & 0 \\
0 & 0 & \ddots & 0 \\
0 & 0 & \cdots & 1 \\
-k_{1}^{i} & -k_{2}^{i} & \cdots & -k_{\sigma_{i}}^{i}
\end{array}\right]}_{\boldsymbol{\Gamma}_{i}} \mathbf{z}_{i} \underbrace{\left[\begin{array}{c}
0 \\
\vdots \\
\lambda_{i}(\mathbf{x})
\end{array}\right]}_{\boldsymbol{\Lambda}_{i}(\mathbf{x})} \mathbf{e}+\underbrace{\left[\begin{array}{c}
0 \\
L_{s} L_{f} h_{i}(\mathbf{x}) \\
\vdots \\
\lambda_{i}(\mathbf{x})+L_{s} L_{f}^{\sigma_{i}-1} h_{i}(\mathbf{x})
\end{array}\right]}_{\mathbf{\Xi}_{i}(\mathbf{x})} \mathbf{d}
$$

where $i=\{1,2\}$, representing the airspeed channel and height channel, respectively.

The property of the output from each subsystem can be established by verifying that the following relation holds:

$$
\mathbf{C}_{i} \boldsymbol{\Gamma}_{i}^{-1} \boldsymbol{\Xi}_{i}(\mathbf{x})=0, \quad \forall i \in\{1,2\}
$$

where $\mathbf{C}_{1}=\left[\begin{array}{lll}1 & 0 & 0\end{array}\right]$ and $\mathbf{C}_{2}=\left[\begin{array}{llll}1 & 0 & 0 & 0\end{array}\right]$. By collecting the first row in (40) and using the relation in (4⿴囗十), the tracking errors for airspeed and height, i.e. $z_{1}^{1}$ and $z_{1}^{2}$, can be further rewritten as

$$
\begin{aligned}
z_{1}^{i} & =\mathbf{C}_{i} \boldsymbol{\Gamma}_{i}^{-1}\left[\dot{\mathbf{z}}_{i}-\boldsymbol{\Lambda}_{i} \mathbf{e}-\boldsymbol{\Xi}_{i} \mathbf{d}\right] \\
& =\mathbf{C}_{i} \boldsymbol{\Gamma}_{i}^{-1}\left[\dot{\mathbf{z}}_{i}-\boldsymbol{\Lambda}_{i} \mathbf{e}\right]
\end{aligned}
$$


It can be seen from (42) that the disturbance terms are decoupled from the output channels in an asymptotic manner. This means that if the system state reaches to a bounded steady-state value, i.e. $\dot{\mathbf{z}}_{i}=0$, and the disturbance estimation error e approaches zero, the tracking error $z_{1}^{i}=0$. Moreover, given that the derivatives of the disturbance are bounded, the closed-loop system can be proved to be locally input-to-state stable under mild conditions as exemplified in [106, [25].

The structure of the UAV dynamics under the composite controller (\$7) is illustrated in Fig.[2. One feature of the proposed control structure is that in the absence of disturbances, the composite control law (B7) reverts to the baseline control law so that the nominal control performance can be recovered.

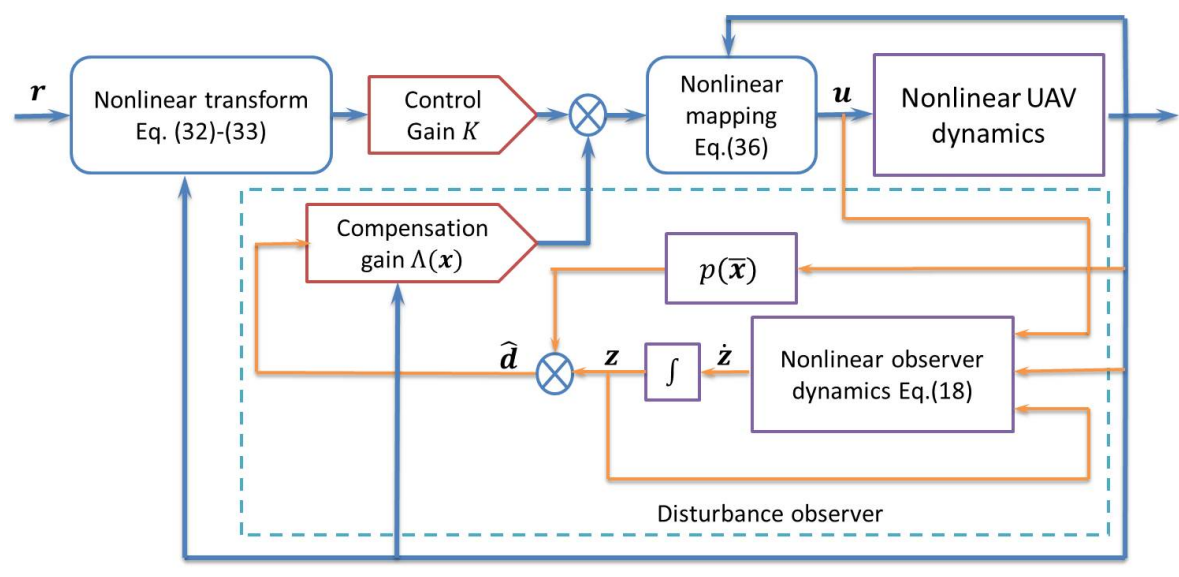

Fig. 2 Control system structure

\section{Simulation study}

To demonstrate the performance of the proposed disturbance rejection control, a number of simulation studies are carried out based on a representative nonlinear model of a small UAV from [20]. The parameters of this UAV are given in the Nomenclature. To include wind influences, a discrete wind gust model following the Military Specification MIL-F-8785C [Z27] is adopted in the simulation, which represents a discrete wind shear such as the fluctuations due to the wake of a 
building. The mathematical representation of this model can be written as:

$$
V_{\text {wind }}=\left\{\begin{array}{cc}
0, & x_{v}<0 \\
\frac{V_{m}}{2}\left(1-\cos \left(\frac{\pi x_{v}}{d_{m}}\right)\right) & 0 \leq x_{v} \leq d_{m} \\
V_{m} & x_{v}>d_{m}
\end{array}\right.
$$

where $V_{\text {wind }}$ is current gust value, $V_{m}$ is the gust amplitude, $d_{m}$ is the gust length and $x_{v}$ is the relative position of the UAV. This gust model can be applied to each axis individually, or to all three axes at once. In the simulation, a sequence of multiple gusts with different starting time and wind parameters are employed.

In realizing the proposed control scheme, the baseline controller can be designed based on the transformed linear system using linear control techniques. In this work, the Linear Quadratic Regulator (LQR) method is used to generate the following control gain matrix:

$$
\mathbf{K}=\left[\begin{array}{ccccccc}
10.00 & 42.4 & 39.8 & 0 & 0 & 0 & 0 \\
0 & 0 & 0 & 15.8 & 78.0 & 113.3 & 56.8
\end{array}\right]
$$

Given the baseline control gain, the disturbance compensation effort can be constructed by feeding the disturbance information via (ㅍ) and (피). In disturbance observer design, the gain parameters $l_{i}$, are chosen as $l_{1}=l_{2}=2, l_{3}=l_{4}=5$ and $l_{5}=l_{6}=5$, which can guarantee a good convergence rate of the disturbance estimates.

To show the effectiveness of the proposed control scheme in rejecting disturbances and improving the robustness, two case studies are carried out. The performance of the composite controller is compared with the baseline NDI controller and another NDI design with integral action, which is a traditional way of resolving the steady-state error. The integral gain used in the simulation was carefully tuned to achieve a good trade-off between the convergence rate and robustness.

\section{A. Disturbance rejection performance}

In the first case study, the UAV is controlled to track a landing profile with a constant airspeed at $25 \mathrm{~m} / \mathrm{s}$ and descending height from $100 \mathrm{~m}$ to $0 \mathrm{~m}$. Therefore, the precise control of airspeed and height under wind conditions is critical for UAV safety. In addition to constant disturbances $d_{D}=0.2 \mathrm{~m} / \mathrm{s}^{2}$, $d_{L}=-0.2 \mathrm{~m} / \mathrm{s}^{2}$ and $d_{M}=0.5 \mathrm{rad} / \mathrm{s}^{2}$, the external wind components applied on the UAV dynamics 
include the wind speed $\left(w_{x}, w_{h}\right)$ and accelerations $\left(\dot{w}_{x}, \dot{w}_{h}\right)$. The disturbance signals together with their estimates from the proposed disturbance observer are illustrated in Fig.31-4. It can be seen that although small gains are used in the observer design, the estimates can quickly converge to the true values of disturbances.

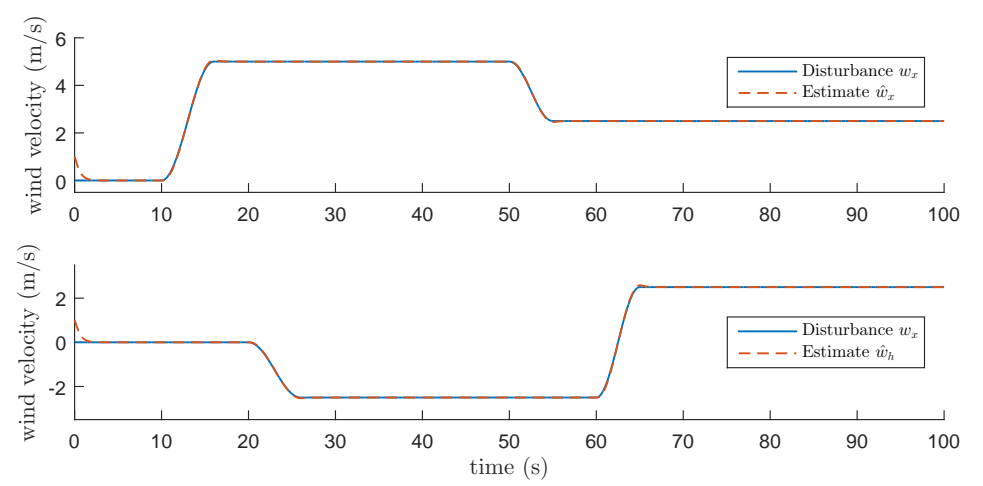

Fig. 3 Wind speed disturbances and their estimates

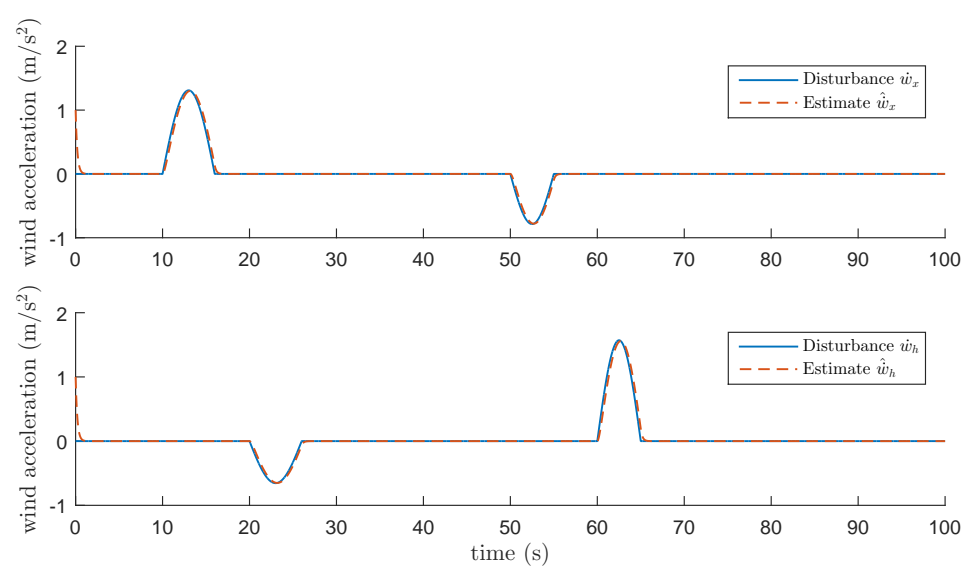

Fig. 4 Wind acceleration disturbances and their estimates

The tracking performance of different control methods under the above disturbances are summarized in Fig. [1] and Fig.[6 for height and airspeed commands, respectively. It can be easily observed that the proposed DOBC method outperforms the other two control methods in terms of the tracking accuracy. The baseline NDI control is able to stabilize the nonlinear dynamics of the UAV, but with larger steady-state errors in the presence of disturbances. The NDI control with integral action can compensate the disturbances to some extent. However, it exhibits overshoot when the reference signal or disturbances changes. These phenomena are not desirable, especially when tracking a 
landing profile. The proposed composite control, on the other hand, is able to exploit the estimated disturbance information to form an active compensation control effort. Therefore, it demonstrates a much improved disturbance rejection capability with a better tracking accuracy.

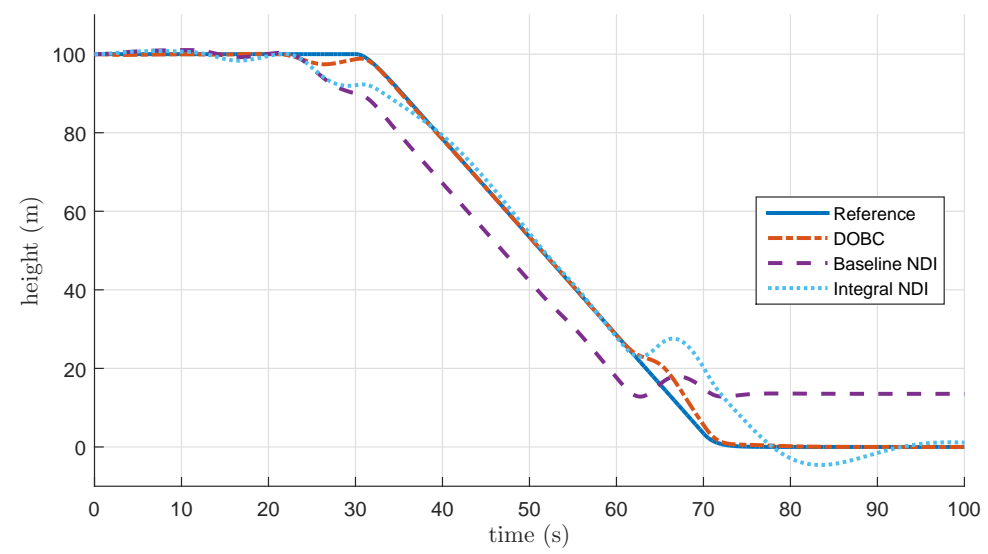

Fig. 5 Height tracking performance

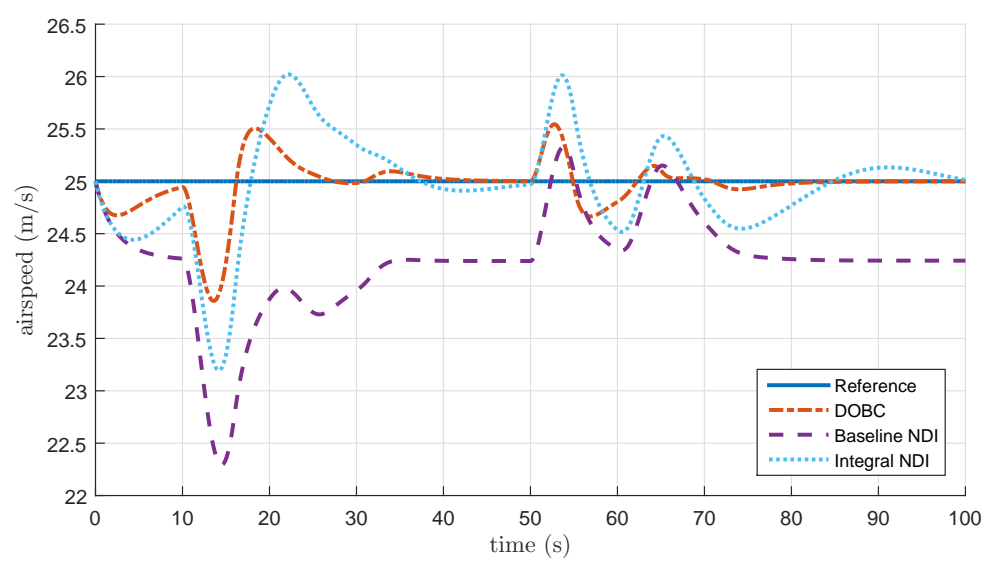

Fig. 6 Airspeed tracking performance

\section{B. Robustness improvement against parameter uncertainties}

The second case study focuses on the robustness of the composite controller. Therefore, the inertial and aerodynamics parameters are assumed to have some uncertainties in the simulation as

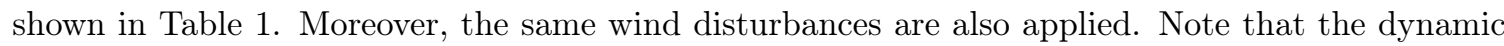
uncertainties do not affect the kinematics (미) directly, but will be coupled with wind acceleration disturbances $\left(\dot{w}_{x}, \dot{w}_{h}\right)$ in other channels. 


\begin{tabular}{c|c|c|c}
\hline \hline Parameters & Uncertainty (\%) & Parameters & Uncertainty (\%) \\
\hline$m, I_{y y}, C_{L_{\alpha}}, C_{m_{\alpha}}$ & 25 & $C_{L_{0}}, C_{L_{\delta_{e}}}$ & 10 \\
\hline$S, b, c, K_{\text {motor }}$ & -10 & $C_{m_{0}}, C_{m_{q}}, C_{m_{\delta_{e}}}$ & -20 \\
\hline
\end{tabular}

The simulation scenario requires the UAV to reduce the airspeed from $35 \mathrm{~m} / \mathrm{s}$ to $25 \mathrm{~m} / \mathrm{s}$ while maintaining a constant height at $100 \mathrm{~m}$. Since the airspeed spans a large range across the flight envelope, the nonlinearity of the dynamics will be excited. The tracking results of this case study are given in Fig. $\boldsymbol{\square}$ and $\mathbf{\nabla}$, which manifest that the controller design based on the NDI technique is able to deal with the nonlinear dynamics and provide stability. However, the baseline NDI controller in this scenario demonstrates notable tracking errors especially on the height channel due to the combination of the external disturbance and internal uncertainties. The NDI controller with integral action gives a better tracking accuracy than the baseline controller. Nevertheless, compared to DOBC it yields a more oscillatory outputs and its correction is much slower as it needs the error signals to accumulate adequately to take actions. This becomes more obvious at $60 \mathrm{~s}$ of the simulation when the vertical wind speed starts to change from $-2 \mathrm{~m} / \mathrm{s}$ to $2 \mathrm{~m} / \mathrm{s}$. The corresponding height output under the integral NDI control results in a large deviation from the reference signal, whereas the output under DOBC quickly recovers to the reference signal because it exploits the estimated disturbances in a feed-forward fashion.

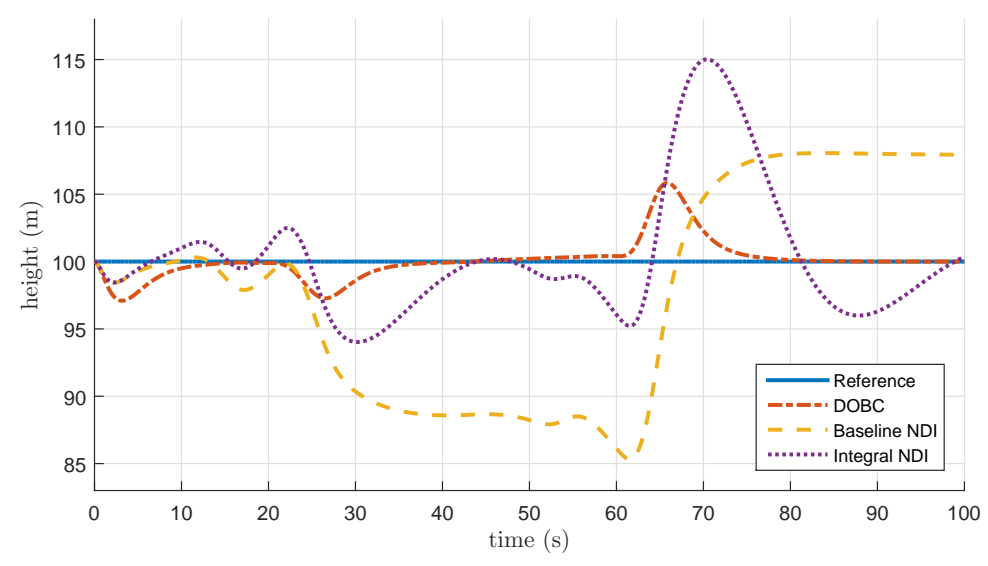

Fig. 7 Height tracking performance against uncertainties 


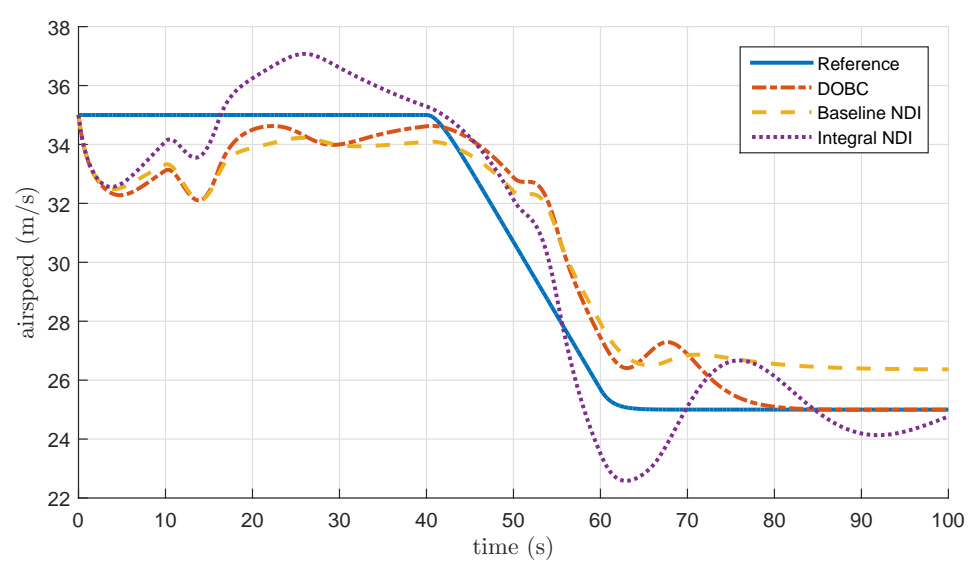

Fig. 8 Airspeed tracking performance against uncertainties

In addition to the control performance, some applications may also be interested in wind estimation under dynamics uncertainties. To this end, estimate of wind accelerations are listed in Fig.9. It can be observed from the results that the estimation performs with a good accuracy and is not affected by dynamics uncertainties. This is because in the proposed observer design, their influences are taken into account by the lumped disturbance $d_{D}$ and $d_{L}$, thus they are separated from wind acceleration terms. The corresponding estimates on $d_{D}$ and $d_{L}$ are shown in Fig.س.

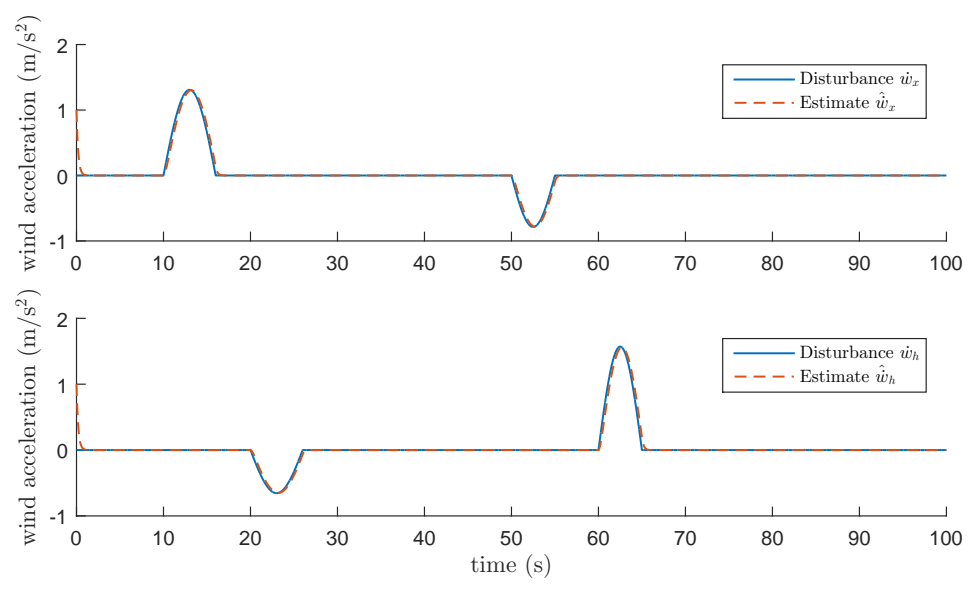

Fig. 9 Wind acceleration disturbances and their estimates

\section{Conclusion}

In this work, a novel disturbance observer based control scheme is developed for the longitudinal flight dynamics of a small fixed-wing unmanned aerial vehicle in order to improve its flight 


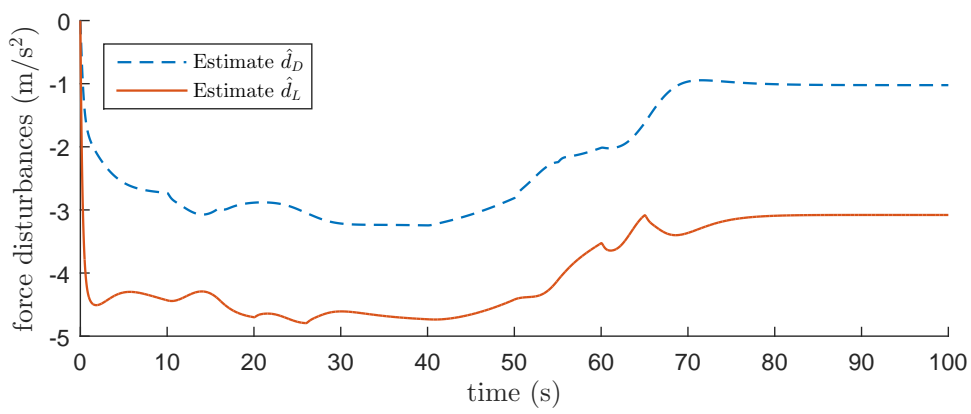

Fig. 10 Estimation on lumped force disturbances

performance in the presence of wind disturbances. This control scheme uses nonliner dynamics inversion to address the nonlinearities in the flight dynamics so that it can follow the reference commands in airspeed and height. A novel nonlinear disturbance observer is designed based on the nominal aircraft model to provide estimates of the wind influences and system uncertainties. These estimates are then used to form the compensation control efforts. The developed scheme has been tested in the simulation studies, with scenarios of landing profile tracking and straight flight under wind disturbances and parameter uncertainties. The results have shown a significant improvement in control accuracy and robustness comparing to the baseline controllers and the controller with integral actions. It should be noted that although the current disturbance observer solution is tailored to the particular problem described in this note, this method may be of interest in many similar flight control applications for different aircraft.

\section{References}

[1] Watkins, S., Milbank, J., Loxton, B. J., and Melbourne, W. H., "Atmospheric winds and their implications for microair vehicles," AIAA journal, Vol. 44, No. 11, 2006, pp. 2591-2600. doi:10.2514/1.22670.

[2] Rysdyk, R., "Unmanned aerial vehicle path following for target observation in wind," Journal of guidance, control, and dynamics, Vol. 29, No. 5, 2006, pp. 1092-1100. doi:10.2514/1.19101.

[3] Liu, C., McAree, O., and Chen, W.-H., "Path-following control for small fixed-wing unmanned aerial vehicles under wind disturbances," International Journal of Robust and Nonlinear Control, Vol. 23, No. 15, 2013, pp. 1682-1698. doi:10.1002/rnc.2938.

[4] Sarras, I. and Siguerdidjane, H., "On the guidance of a UAV under unknown wind disturbances," Control Applications (CCA), 2014 IEEE Conference on, Oct 2014, pp. 820-825. 


\section{doi:10.1109/CCA.2014.6981442.}

[5] Hervas, J. R., Reyhanoglu, M., Tang, H., and Kayacan, E., "Nonlinear control of fixed-wing UAVs in presence of stochastic winds," Communications in Nonlinear Science and Numerical Simulation, Vol. 33, 2016, pp. 57 - 69. doi:10.1016/j.cnsns.2015.08.026.

[6] Okamoto, K. and Tsuchiya, T., "Optimal Aircraft Control in Stochastic Severe Weather Conditions," Journal of Guidance, Control, and Dynamics, 2015. doi:doi: 10.2514/1.G001105.

[7] Liao, F., Wang, J. L., Poh, E. K., and Li, D., "Fault-tolerant robust automatic landing control design," Journal of guidance, control, and dynamics, Vol. 28, No. 5, 2005, pp. 854-871. doi:10.2514/1.12611.

[8] Wang, Q. and Stengel, R. F., "Robust nonlinear control of a hypersonic aircraft," Journal of Guidance, Control, and Dynamics, Vol. 23, No. 4, 2000, pp. 577-585. doi:10.2514/6.1999-4000.

[9] Xu, H., Mirmirani, M. D., and Ioannou, P. A., "Adaptive sliding mode control design for a hypersonic flight vehicle," Journal of guidance, control, and dynamics, Vol. 27, No. 5, 2004, pp. 829-838. doi: $10.2514 / 1.12596$.

[10] Fiorentini, L., Serrani, A., Bolender, M. A., and Doman, D. B., "Nonlinear robust adaptive control of flexible air-breathing hypersonic vehicles," Journal of guidance, control, and dynamics, Vol. 32, No. 2, 2009, pp. 402-417. doi:10.2514/1.39210.

[11] Fiorentini, L. and Serrani, A., "Adaptive restricted trajectory tracking for a non-minimum phase hypersonic vehicle model," Automatica, Vol. 48, No. 7, 2012, pp. 1248 - 1261. doi:10.1016/j.automatica.2012.04.006.

[12] Gavilan, F., Vazquez, R., and Acosta, J. A., "Adaptive Control for Aircraft Longitudinal Dynamics with Thrust Saturation," Journal of guidance, control, and dynamics, Vol. 38, No. 4, 2015, pp. 651661. doi:10.2514/1.G000028.

[13] Li, S., Yang, J., Chen, W., and Chen, X., Disturbance Observer-Based Control: Methods and Applications, CRC Press, 2014.

[14] Chen, W.-H., "Nonlinear disturbance observer-enhanced dynamic inversion control of missiles," Journal of Guidance, Control, and Dynamics, Vol. 26, No. 1, 2003, pp. 161-166. doi:10.2514/2.5027.

[15] Zhang, Z., Li, S., and Luo, S., "Terminal guidance laws of missile based on ISMC and NDOB with impact angle constraint," Aerospace Science and Technology, Vol. 31, No. 1, 2013, pp. 30 - 41. doi:10.1016/j.ast.2013.09.003.

[16] Yang, J., Zhao, Z., Li, S., and Zheng, W. X., "Composite predictive flight control for airbreathing hypersonic vehicles," International Journal of Control, Vol. 87, No. 9, 2014, pp. 1970-1984. doi:10.1080/00207179.2014.896477. 
[17] Liu, C., Chen, W.-H., and Andrews, J., "Tracking control of small-scale helicopters using explicit nonlinear MPC augmented with disturbance observers," Control Engineering Practice, Vol. 20, No. 3, 2012, pp. 258 - 268. doi:10.1016/j.conengprac.2011.10.015.

[18] Lu, H., Liu, C., Guo, L., and Chen, W.-H., "Flight Control Design for Small-Scale Helicopter Using Disturbance-Observer-Based Backstepping," Journal of Guidance, Control, and Dynamics, Vol. 38, No. 11, 2015, pp. 2235-2240. doi:10.2514/1.G001196.

[19] Mulgund, S. S. and Stengel, R. F., "Optimal nonlinear estimation for aircraft flight control in wind shear," Automatica, Vol. 32, No. 1, 1996, pp. 3 - 13. doi:10.1016/0005-1098(95)00102-6.

[20] Pourtakdoust, S. H., Kiani, M., and Hassanpour, A., "Optimal trajectory planning for flight through microburst wind shears," Aerospace Science and Technology, Vol. 15, No. 7, 2011, pp. 567 - 576. doi:10.1016/j.ast.2010.11.002.

[21] Serrani, A., Isidori, A., and Marconi, L., "Semi-global nonlinear output regulation with adaptive internal model," IEEE Transactions on Automatic Control, Vol. 46, No. 8, Aug 2001, pp. 1178-1194. doi:10.1109/9.940923.

[22] Chen, W.-H., "Disturbance observer based control for nonlinear systems," Mechatronics, IEEE/ASME Transactions on, Vol. 9, No. 4, Dec 2004, pp. 706-710. doi:10.1109/TMECH.2004.839034.

[23] Wu, H.-N., Liu, Z.-Y., and Guo, L., "Robust $L_{\infty}$-Gain Fuzzy Disturbance Observer-Based Control Design With Adaptive Bounding for a Hypersonic Vehicle," IEEE Transactions on Fuzzy Systems, Vol. 22, No. 6, Dec 2014, pp. 1401-1412. doi:10.1109/TFUZZ.2013.2292976.

[24] Isidori, A., Nonlinear Control Systems II, Communications and Control Engineering, Springer London, 1999, Chapter 10.

[25] Yang, J., Li, S., and Chen, W.-H., "Nonlinear disturbance observer-based control for multi-input multioutput nonlinear systems subject to mismatching condition," International Journal of Control, Vol. 85, No. 8, 2012, pp. 1071-1082. doi:10.1080/00207179.2012.675520.

[26] Beard, R. and McLain, T., Small Unmanned Aircraft: Theory and Practice, Princeton University Press, 2012, Page 276.

[27] Moorhouse, D. J. and Woodcock, R. J., "Background information and user guide for MIL-F-8785C, military specification-flying qualities of piloted airplanes," DTIC report AFWAL-TR-81-3109, July 1982. 